\section{A comparison of induced astigmatism in 20-vs 25-gauge vitrectomy procedures}

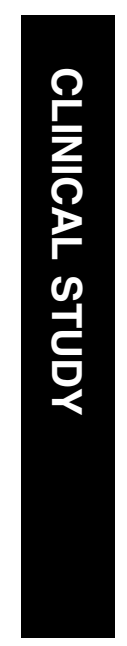

refractive outcome for the patient.

Eye (2010) 24, 315-317; doi:10.1038/eye.2009.81;

published online 24 April 2009

Keywords: vitrectomy; induced astigmatism; sutureless; keratometry; 25-gauge; pars plana vitrectomy; 20-gauge pars plana vitrectomy

\section{Introduction}

With the advent of smaller microsurgical instruments, the 25-gauge 'sutureless' pars plana vitrectomy has rapidly been adopted as the preferred technique for vitreoretinal surgery. Currently, recognised advantages of the 25-gauge procedure include less postoperative pain and shorter operating times. ${ }^{1}$ This study aims to investigate the effect of both 20- gauge and 25-gauge vitrectomies on corneal curvature through a comparison of the differences in pre- and post-surgery keratometry readings. Post-vitrectomy astigmatism is a wellrecognised consequence of vitrectomy. ${ }^{2-5}$ The ability to minimise this surgically induced astigmatism (SIA) assists in achieving optimal refractive and visual outcomes for patients.

\section{Materials and methods}

The study is a retrospective, non-randomised consecutive case series of vitrectomies performed by a single surgeon (RDB). The study population consisted of 47 patients, eight of whom had bilateral procedures. The indications for vitrectomy included macular hole repair, epiretinal membrane peeling, and radial optic neurotomy. Twenty-four procedures were performed using the 20-gauge technique and 31 using the 25-gauge procedure. Exclusion criteria included; pathology altering corneal
Department of Vitreoretinal Surgery, Vision Retinal Institute, Varsity Lakes, Queensland, Australia

Correspondence: B Drury, Department of Optometry, Visions By Brian Job Optical, 1/1 Stanley Street,

Tweed Heads,

New South Wales 2485

Australia

Tel: + 0407218 698;

Fax: + 61755312016 .

E-mail: brett.drury1@ gmail.com

Received: 14 July 2008 Accepted in revised form: 17 March 2009 Published online: 24 April 2009

Proprietary interests: None

Presented at the ASCRS Symposium on Cataract, IOL and Refractive Surgery 2005 Washington 
topography such as keratoconus, scleral buckling procedure, and the absence of pre- and post-vitrectomy keratometry.

Both the 20- and 25-gauge procedures were performed using a three-port pars plana technique: one irrigation port in the inferotemporal quadrant, and two instrument ports: superonasal and superotemporal. Twenty gauge procedures used instruments with a diameter of $0.813 \mathrm{~mm}$ and a cross-sectional area of $0.519 \mathrm{~mm}^{2}$, the sclerotomy sites were sutured using 8.0 vicryl. The 25-gauge technique used a trochar diameter of $0.519 \mathrm{~mm}$ with a cross-sectional area of $0.2026 \mathrm{~mm}^{2}$, the conjunctiva was caressed over the sclerotomy sites to assist in self-sealing; no sutures were used.

At presentation, all patients were phakic in the affected eye. This population was chosen as they all had postvitrectomy keratometry readings recorded as part of prePHACO and IOL insertion investigations. Measurements were made using a manual keratometer, IOL Master, or Humphrey Autorefractor. Refractive values were not used as these include a component of lenticular astigmatism, an indeterminable variable. ${ }^{6,7}$

Vector analysis of pre- and post-vitrectomy readings was performed using Alpin's method, facilitated by the ASSORT program version 4.1. ${ }^{8}$ All investigation was conducted in accordance with the Declaration of Helsinki of 1975.

\section{Results}

Postoperative keratometry readings were taken at mean of 3.9 months (1-36), with no statistically significant difference between follow-up for 20- and 25-gauge procedures.

Table 1 shows that the 25-gauge vitrectomy produces less astigmatism than the 20-gauge technique. The mean SIA for the 25-gauge procedure (0.27 D) was significantly less $(P=0.037)$ than that for the 20-gauge procedure $(0.66 \mathrm{D})$. The SD in SIA variation in the 25-gauge group was less than one-third of that in the 20-gauge group (0.73 vs 2.28).

Table 1 Comparison of 20- vs 25-gauge results (SIA)

\begin{tabular}{lcc}
\hline & 20 gauge & 25 gauge \\
\hline Sample size & 24 & 31 \\
Mean presenting astigmatism & $0.63 \mathrm{D}$ & $0.92 \mathrm{D}$ \\
Mean post-surgical astigmatism & $1.14 \mathrm{D}$ & $0.91 \mathrm{D}$ \\
Mean K2 angle at presentation & $93.65^{\circ}$ & $93.13^{\circ}$ \\
Mean K2 angle post-surgery & $94.17^{\circ}$ & $94.86^{\circ}$ \\
Mean SIA $(P=0.037)$ & $0.66 \mathrm{D}$ & $0.27 \mathrm{D}$ \\
SD of SIA & $0.80 \mathrm{D}$ & $0.23 \mathrm{D}$ \\
SIA variability $(95 \% \mathrm{CI})$ & $2.26 \mathrm{D}$ & $0.73 \mathrm{D}$ \\
\hline
\end{tabular}

Abbreviation: SIA=surgically induced astigmatism.

\section{Discussion}

The magnitude of the difference in SIA between the 20- and 25-gauge groups is both statistically and may also be clinically significant considering the pathological indications for which the vitrectomies were undertaken. Through modern surgical techniques, visual outcomes in patients suffering from macular holes and epiretinal membranes are now exceptional. Kumagai et al, ${ }^{9}$ performed a long-term follow-up of 190 eyes who underwent macular hole repair (stage 2-4 macular holes) and found an average visual acuity of $0.12 \log$ MAR, with $44 \%$ of patients achieving visual acuity $\geqslant 6 / 6$ and $86 \%$ achieving $\geqslant 6 / 12$. Epiretinal membrane peeling shows similarly successful visual outcomes, with reported mean visual improvement of $0.3 \log$ MAR and mean postoperative visual acuity of $6 / 12 .{ }^{10}$ At these high levels of visual acuity, noticeable blur limits are much smaller ${ }^{11}$ and consequently a SIA of $0.27 \mathrm{D}$ is expected to go unnoticed by the patient whereas $0.66 \mathrm{D}$ may be visually detectable, ${ }^{12}$ possibly requiring a new spectacle prescription.

The precision of the refractive outcome is another important factor to consider when interpreting the findings of this study. The SD and 95\% CI for the SIA in the 20- and 25-gauge groups were 0.8 and $2.28 \mathrm{D}$ and 0.23 and $0.73 \mathrm{D}$, respectively. The 25 -gauge group showed a far more precise and thus predictable amount of SIA than the 20-gauge group. This highlights yet another advantage of the 25-gauge procedure, as the surgeon can be assured that they are far less likely to induce large, unfavourable amounts of astigmatism.

Surgically induced astigmatism detracts from the visual acuity outcome of pseudophakic patients more than that of phakic patients. Given that almost all phakic patients undergoing vitrectomy will subsequently develop a cataract as procedural complication, ${ }^{13,14}$ the resultant IOL implantation procedure provides an opportunity for correction of any SIA. Unfortunately, for the pseudophakic patient, any subsequent intervention to correct SIA will be an additional surgical procedure for that purpose only. Thus, in the absence of a corrective procedure, pseudophakic patients undergoing vitrectomy must bear any resultant astigmatism as an unfortunate consequence. Hence, a vitrectomy technique that minimises SIA provides a superior visual and refractive outcome, particularly for pseudophakic patients.

Although the sample size used in this study is commensurate with, and in most cases significantly greater than, the very few other studies in the area, this series does have some limitations. These relate to the inexact axis of port sites, absence of sequential keratometry readings, variable timing of keratometry readings, and the use of multiple varieties of keratometers. 
The variable timing of post-vitrectomy keratometry measurements bears significance predominantly for the 20-gauge group, where vicryl sutures were used. These sutures take 6 weeks to dissolve and may affect corneal topography during this period. The average timing of post-vitrectomy keratometry measurements was 3.9 months, however, in the 20-gauge group only one patient had their keratometry readings recorded within 6 weeks of vitrectomy. The impact of this single reading on the data and conclusions drawn would be negligible.

The use of multiple keratometers to measure the corneal curvatures between preoperative and post-operative visits is a shortcoming of this study and is unfortunately an unavoidable consequence of conducting retrospective research. The impact of this confounder on the significance of the results presented here is debatable. Older studies have shown no significant difference between automated (portable and fixed) and manual keratometers, ${ }^{15,16}$ whereas more recent authors have suggested the instruments may not be interchangeable. ${ }^{17}$

\section{Conclusions}

This retrospective study shows another advantage of the 25-gauge vitrectomy procedure over the 20-gauge procedure. The 25-gauge vitrectomy produces significantly less SIA and gives a more predictable and precise refractive result. If vitrectomy induced astigmatism can be minimised, additional refractive corrections are redundant. This difference is especially pertinent in patients who are likely to achieve a good visual outcome after the vitrectomy and those who are pseudophakic preoperatively.

\section{References}

1 Ibarra M, Hermel M, Prenner J, Hassan T. Longer-term outcomes of transconjunctival sutureless 25-gauge vitrectomy. Am J Ophthalmol 2005; 139: 831-836.
2 Randleman J, Hewitt S, Stulting R. Refractive changes after posterior segment surgery. Ophthalmol Clin North Am 2004; 17: 521-vi.

3 Sinha R, Sharma N, Verma L. Corneal topographic changes following retinal surgery. BMC Ophthalmol 2004; 4: 10.

4 Weinberger D, Lichter H, Loya N, Axer-Siegel R, Muzmacher L, Gabbay U et al. Corneal topographic changes after retinal and vitreous surgery. Ophthalmology 1990; 106(8): 1521-1524.

5 Wirbelauer C, Hoerauf H, Roider J, Laqua H. Corneal shape changes after pars plana vitrectomy. Graefes Arch Clin Exp Ophthalmol 1998; 236: 822-828.

6 Dashevskii A, Kuzina E. Clinical aspects of vector analysis of lenticular astigmatism. Oftamol Zh 1987; 11: 70-72.

7 Liang D, Guan Z, Lin J. The relations of corneal, lenticular and total astigmatism. Yan Ke Хue Bao 1995; 11: 70-72.

8 Jaffe N, Jaffe G, Jaffe M. Postoperative Corneal Astigmatism. Mosby: St Louis, 1997.

9 Kumagai K, Furukawa M, Ogino N, Uemura A, Larson E. Long-term outcomes of internal limiting membrane peeling with and without indocyanine green in macular hole surgery. Retina 2006; 26: 613-617.

10 Wong J, Sachdev N, Beaumont P, Chang A. Visual outcomes following vitrectomy and peeling of epiretinal membrane. Clin Experiment Ophthalmol 2005; 33: 373-378.

11 Atchinson D, Fisher S, Pederson C, Ridall P. Noticable, troublesome and objectionable limits of blur. Vision Res 2005; 45: 1967-1974.

12 Miller A, Kris M, Griffiths A. Effect of small focal errors on vision. Optom Vis Sci 1997; 74(7): 521-526.

13 Blodi B, Paluska S. Cataract after vitrectomy in young patients. Ophthalmology 1997; 104: 1092-1095.

14 Thompson J. The role of patient age and intraocular gas use in cataract progression after vitrectomy for macular holes and epiretinal membranes. Am J Ophthalmol 2004; 137: 250-257.

15 Knorz M, Kohl M, Trinkmann R. Automatic biometry and keratometry in comparison with the manual technic Fortschr Ophthalmol 1989; 86(2): 157-158.

16 Manning C, Kloess P. Comparison of portable automated keratometry and manual keratometry for IOL calculation. J Cataract Refract Surg 1997; 23(8): 1213-1216.

17 Elbaz U, Barkana Y, Gerber Y, Avni I, Zadok D. Comparison of different techniques of anterior chamber depth and keratometric measurements. Am J Ophthalmol 2007; 143(1): $48-53$. 